\title{
Biomechanical factors associated with the risk of knee injury when landing from a jump
}

\author{
Q Louw (PhD) ${ }^{1}$ \\ K Grimmer (PhD) $)^{2}$ \\ 'Department of Physiotherapy, Stellenbosch University, W Cape \\ ${ }^{2}$ Centre of Allied Health Evidence, University of South Australia
}

\begin{abstract}
Objectives. To systematically assess the literature investigating biomechanical knee injury risk factors when an individual lands from a jump.

Data sources. Four electronic databases were searched for peer-reviewed English journals containing landing biomechanical studies published over 14 years (1990 - 2003).

Study selection. Publications describing research into knee joint kinetics and/or kinematics when landing from a jump were included. A total of 26 eligible articles met the
\end{abstract} inclusion criteria.

Data extraction. A review of the 26 eligible studies was undertaken to describe the key study components including the study aims, sample populations, measurement tools, measurement procedures and knee risk factors. Methodological quality was scored using the Crombie Checklist and PEDro Scale.

Data synthesis. The methodological quality of the studies reviewed was fair. Information on risk factors was variable. One proposed risk factor, landing with the knee in a relatively more extended position, may increase injury risk. Validity was compromised when the landing action was isolated by studying drop-jumping instead of the whole landing task. Results of reviewed studies were potentially confounded by a number of factors.

Conclusion. High-level evidence for biomechanical knee injury risk factors when landing from a jump is lacking and it is difficult to draw conclusions regarding knee injury risk factors when landing. However, the published research reviewed provides important information on injury causality and theories to direct future studies. Further research should be directed towards younger populations using valid testing protocols applicable to real life scenarios.

\section{CORRESPONDENCE:}

\section{Q Louw}

Department of Physiotherapy

Stellenbosch University

PO Box 19063

Tel: 021-938 9300

Fax: 021-931 1252

E-mail: qalouw@sun.ac.za
Landing badly from a jump during sporting activities is one of the common injury mechanisms leading to lower limb injuries. ${ }^{24}$ Landing badly refers inadequate lower limb control resulting in torsion of the knee or falling which may strain the knee soft tissue structures and lead to injury. Identifying and understanding biomechanical risk factors when a player lands from a jump could aid in the development of preventive programmes. ${ }^{40}$ Reportedly basketball has the highest rate of serious knee injuries among non-contact sports. ${ }^{18}$ Landing badly often results in more serious knee injuries measured by time lost from play or medical management. 18,25 Competitive adolescent basketball players in Cape Town reported that $41 \%$ of the knee injuries sustained over one season were related to landing badly from a jump..$^{24}$ This finding concurs with similar international studies. ${ }^{18,25,39}$

Landing is a complex action and requires the player to coordinate upper body, trunk and lower limb movements, absorb forces imposed on the body and maintain hip, knee and ankle stability during the movement execution. ${ }^{23}$ Joint stability refers to a joint remaining or returning to proper alignment through equalisation of forces in the presence of forces that would normally change the state or condition of the joint and is critical in preventing injury to the knee joint structures. ${ }^{26,35}$ Static joint stabilisers include the ligaments, joint capsules, cartilage, bone and joint shape. ${ }^{21}$ Dynamic joint stability stems from neuromuscular control mechanisms. ${ }^{21}$ Injury occurs to the static and dynamic restraints when the forces imposed on these systems exceed the mechanical threshold. ${ }^{21}$ When a player lands from a jump, biomechanical factors that could compromise joint stability include ground reaction forces, joint position or angles, joint moments and work.

The aim of this systematic review was to systematically assess the literature reporting on the biomechanical knee injury risk factors when an individual lands from a jump. This review also evaluated what has been written on landing techniques in order to identify shortfalls in the published literature that could be applied in future landing analysis studies. The objectives of this review were to: (i) appraise information from biomechanical studies of landing published from 1990 to 2003; (ii) appraise methodological rigor of the biomechanical studies of landing published from 1990 to 2003; (iii) review the testing protocol employed in published studies; (iv) ascertain the populations commonly included in biomechanical landing studies; ( $v$ ) review risk factors identified by previous studies; and ( $v i)$ identify shortcomings of published studies of landing biomechanics. 


\section{Methodology}

\section{Level of evidence}

The hierarchical system of research design evidence as described by Sackett et al. ${ }^{36}$ was used to determine the levels of research evidence in the published literature.

\section{Search strategy}

Databases available at the University of South Australia were searched for peer-reviewed journals with papers on landing biomechanical studies. These databases included Medline, Cinahl, Current Contents and Sport Discus.

The following key words were used: (i) landing biomechanics; (ii) landing and jump; (iii) jump landing and biomechanics; (iv) forces, landing and jump; (v) kinematics and/or kinetics, landing and jump; and ( $V i)$ mechanics, landing and jump.

\section{Inclusion criteria}

Peer-reviewed English language publications since 1990 describing research into knee joint kinetics and/or kinematics when landing from a jump were included. The search was limited from 1990 to 2003 as the last 14 years of literature provided evidence from a decade of research, as well as the most recent research findings.

\section{Exclusion criteria}

Research published prior to 1990 was excluded, unless it was a landmark study (cited by many other authors after 1990). Review papers were excluded. Research describing the biomechanical aspects of internal joint forces was not included in this review. Studies of landing in recreational activities such as rope jumping and general functional activities were also excluded.

\section{Methodological quality}

The Crombie Checklist ${ }^{4}$ was used to appraise the case-control and observational studies and the PEDro Scale was used to appraise randomised controlled trials. ${ }^{32}$ The 11 crite- ria of the PEDro scale included: (i) eligibility criteria; (ii) random allocation of subjects; (iii) concealed allocation of subjects; (iv) prognostic indicators of the groups were similar at baseline; ( $v$ ) blinding of all subjects; ( $v i)$ blinding of all therapists who administered the therapy; (vii) blinding of all assessors who measured at least 1 key outcome; (viii) measures of key outcomes obtained from $85 \%$ of subjects; (ix) all subjects for whom outcome measures were available received the treatment or control condition as allocated; $(x)$ the results of between-group statistical comparisons reported for at least 1 key outcome; and ( $x i)$ the study provides both point measures and measures of variability for at least 1 key outcome.

\section{Results}

\section{Database search results}

The extensive search yielded 920 hits. After removal of duplicate titles and those that did not meet the inclusion criteria, the full text versions of 26 articles were reviewed. The database search results are presented in Table I.

\section{Level of evidence}

According to Sackett's Hierarchy of Evidence 3 papers reported randomised controlled trials ${ }^{28,31,33}$ and thus present level $2 a$ evidence. ${ }^{36}$ Twenty-three (88\%) of the studies present level 3 evidence. Thirteen observational and 10 casecontrol studies were reviewed.

\section{Methodological quality}

The methodological quality of the studies was fair. Table II presents the results of the methodological quality of the randomised controlled studies. The average score of the observational studies was $67 \%$. The average score of the case-control studies was $72 \%$ and the 3 randomised controlled trials had an average score of $55 \%$.

Fig. 1. Graphically summarises the number of case-control and observational studies that met each of the 12 methodological criteria assessed by the Crombie Checklist. None of

\section{TABLE I. Results of search strategy}

\begin{tabular}{|c|c|c|c|c|c|c|c|c|c|c|}
\hline $\begin{array}{l}\text { Search terms } 1-6 \\
\text { Hits }\end{array}$ & 1 & 2 & 3 & 4 & 5 & 6 & Total hits & $\begin{array}{l}\text { Excluded } \\
\text { duplicates }\end{array}$ & $\begin{array}{l}\text { Excluded on } \\
\text { eligibility }\end{array}$ & $\begin{array}{l}\text { No of articles } \\
\text { retained }\end{array}$ \\
\hline Medline & 124 & 81 & 2 & 32 & 18 & 3 & 260 & 27 & 214 & 19 \\
\hline Cinahl & 30 & 29 & 0 & 16 & 13 & 1 & 89 & 26 & 60 & 3 \\
\hline Current Contents & 32 & 79 & 1 & 36 & 18 & 4 & 170 & 26 & 142 & 2 \\
\hline Sport Discus & 237 & 111 & 0 & 37 & 21 & 3 & 401 & 26 & 374 & 1 \\
\hline Reference lists & & & & & & & & & & 1 \\
\hline Total & & & & & & & & & & 26 \\
\hline
\end{tabular}

\section{TABLE II. Appraisal results of randomised trials (scoring tool: PEDro scale)}

\begin{tabular}{|c|c|c|c|c|c|c|c|c|c|c|c|c|}
\hline Criterion & 1 & 2 & 3 & 4 & 5 & 6 & 7 & 8 & 9 & 10 & 11 & Score \\
\hline McNair and Prapavessis ${ }^{27}$ & $\mathrm{Y}$ & $\mathrm{Y}$ & $\mathrm{N}$ & $\mathrm{N}$ & $\mathrm{N}$ & $\mathrm{N}$ & $\mathrm{Y}$ & $\mathrm{Y}$ & $\mathrm{Y}$ & $\mathrm{Y}$ & $\mathrm{Y}$ & $7 / 11$ \\
\hline McNair and Marshall ${ }^{29}$ & $\mathrm{Y}$ & $\mathrm{Y}$ & $\mathrm{N}$ & $\mathrm{N}$ & $\mathrm{N}$ & $\mathrm{N}$ & $\mathrm{N}$ & $\mathrm{Y}$ & $\mathrm{Y}$ & $\mathrm{Y}$ & $\mathrm{Y}$ & $6 / 11$ \\
\hline Onate et al. ${ }^{31}$ & $\mathrm{Y}$ & Y & $\mathrm{N}$ & $\mathrm{N}$ & $\mathrm{N}$ & $\mathrm{N}$ & $\mathrm{N}$ & $\mathrm{N}$ & Y & $\mathrm{Y}$ & Y & $5 / 11$ \\
\hline
\end{tabular}


the studies met criterion 6 which assessed the reliability and validity of the measurements. Criterion 11 assessed whether there could be confounding and none of studies adequately controlled for confounding. Only $8 \%$ of the studies justified the sample size (criterion 3).

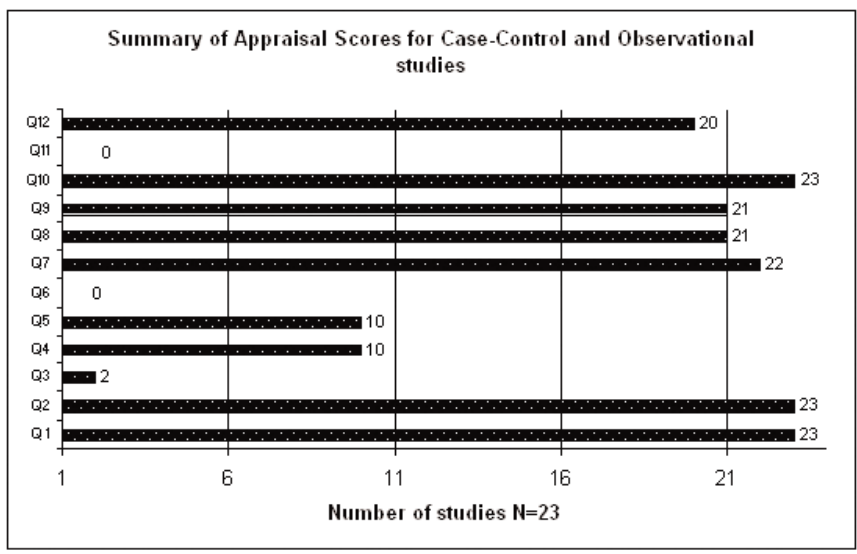

Fig. 1. Summary of appraisal scores.

Aims of published studies and clarity of research questions

The aims of published studies can be divided into 3 categories: (i) analysis of landing technique in uninjured individuals; (ii) factors influencing kinetics and kinematics when landing; and (iii) intra-subject variability of performance.

Eight of the 13 studies published between 1990 and 1999 aimed to analyse landing technique and provide baseline information on landing biomechanics for future studies. ${ }^{1,5,7,8,19,30,38,41}$

Factors influencing lower limb kinetics and kinematics when landing were evaluated by 13 of the 26 studies reviewed. Only 4 of the 13 studies were published before 1999. To summarise the injury risk factors, factors suggested by published studies to reduce knee injury risk were considered 'positive factors' and those factors likely to increase injury risk were considered 'negative factors'. Results of these studies indicated that plyometric training ${ }^{14}$ and augmented verbal feedback positively affect lower limb kinetics and kinematics., ${ }^{3,27}$ Factors found to negatively influence lower limb kinetics and kinematics included female gender, cryotherapy, pubescent stage and injury. $2,5,11,12,13,15,17,22,30,34$

Two published studies on within-participant variability in biomechanical parameters when a subject repeats the same task more than once were reviewed. Both studies were published after the year 2000..$^{16,37}$ This may indicate that intrasubject variability emerged as a factor to consider in injury occurrence from the results of earlier studies. The published studies hypothesised that increased intra-subject variability in biomechanical parameters may reduce the stress placed on a specific joint structure and thereby reduce the chances of injury to that structure due to repeated strain. ${ }^{13}$ Both studies ${ }^{13,37}$ provide level 3 evidence and the findings were inconclusive since the researchers were unable to demonstrate a consistent relationship between intra-subject variability and injury occurrence. Further studies into intra-subject variability and more appropriate statistical methods to analyse the data are required.

The 3 randomised controlled trials aimed to identify mechanisms of reducing ground reaction forces when landing from a jump by using verbal and auditory feedback. . $^{28,31,33}$

\section{Sample descriptions}

The sample sizes of the published studies varied between 3 and 91 participants. Although the mean sample size was 32 , the standard deviation was large (SD 47). Fifteen of the 26 studies reviewed included 20 or less subjects. Seven studies included between 20 and 50 subjects and only 3 studies included more than 50 subjects. Only 2 studies justified the sample size employed in the study. ${ }^{3,15}$

Table III indicates that males were more frequently studied than females. Females were only included in 17 studies, while males were included in 22 studies (Table III). Considering that the prevalence of serious knee injuries such as anterior cruciate injuries is more common among females than males, more studies should include female participants. $^{14}$

Three of the 13 studies including males and females aimed to assess gender differences in lower limb biomechanics when landing. 11,14,22 These studies found that females demonstrated increased lower limb injury risk compared with males. Results of a survey conducted in Cape Town, however, indicated no significant gender difference in sustaining a knee injury between male and female adolescent basketball players (odd's ratio: 1.2, 95\% confidence intervals (Cls): 0.7 - 1.9). ${ }^{24}$

The average age of subjects was 23 years. Three studies included subjects under the age of 20 years. ${ }^{7,14,33}$ The results indicate the lack of information currently available on landing biomechanics among adolescent individuals.

\begin{tabular}{ll}
\multicolumn{2}{l}{ TABLE III. Gender inclusion in published studies } \\
\hline Gender inclusion & No of studies \\
\hline Males only $1,2,8,12,17,19,30,34,41$ & 9 \\
Females only ${ }^{3,5,7,13}$ & 4 \\
Males and females & 13
\end{tabular}

\section{Injury status of published studies}

Six of the 26 studies reviewed included injured participants. Three studies included anterior cruciate ligament (ACL)injured players..$^{5,12,29}$ One study included players who had a history of overuse injury, ${ }^{16} 1$ study included players suffering from patellar tendonitis ${ }^{34}$ and the remaining study included subjects with ankle joint instability. The mean age of subjects included in these studies was 26.5 years (SD 1.8). Currently there is therefore no biomechanical landing information on lower limb kinetics and kinematics of injured adolescents when landing from a jump. Table IV provides a summary of the injury status of participants included in published studies. 
TABLE IV. Injury status of participants in published studies

\begin{tabular}{ll}
\hline & Number of studies \\
\hline Uninjured $^{1,3,5,7,8,11,13-15,17,19,22,28-31,37,38,41}$ & 19 \\
Injured $^{12}$ & 1 \\
Injured and uninjured $^{2,5,16,29,34}$ & 5 \\
Not mentioned $^{27}$ & 1 \\
\hline
\end{tabular}

\section{Type of sporting activity}

Table $\mathrm{V}$ illustrates the type of sports and recreational activity status of samples. Although 9 studies included subjects participating in general jump sports, 3 studies specifically stated that basketball players were included. One study included only basketball players. ${ }^{11}$

\section{TABLE V. Type of sporting activity included in} published studies

\begin{tabular}{|c|c|}
\hline Recreational activity & Number of studies \\
\hline Basketball, volleyball and soccer ${ }^{22}$ & 1 \\
\hline Basketball and volleyball ${ }^{5}$ & 1 \\
\hline Gymnastics $^{30}$ & 1 \\
\hline Basketball ${ }^{11}$ & 1 \\
\hline Jump/landing sports ${ }^{1,5,8,13,16,41}$ & 6 \\
\hline Volleyball 74,14 & 3 \\
\hline No jump sports ${ }^{28}$ & 1 \\
\hline Netball $^{3}$ & 1 \\
\hline General sports $2,12,15,17,19,27,29,31,33,37,38$ & 11 \\
\hline
\end{tabular}

\section{Level of play}

Table VI indicated the 7 terms employed by published studies to describe the level of play. Variations in defining level of play hamper comparison of studies. Thirteen of the 26 studies reviewed used subjects participating only at recreational level and only 3 studies involved only elite athletes (Table VI).

\begin{tabular}{|ll|}
\hline TABLE VI. Level of play & \\
\hline Level of play & No. of studies \\
\hline Elite $^{3,7,34}$ & 3 \\
Elite and recreational $^{30}$ & 1 \\
Recreational $^{1,2,5,8,13,15,16,22,28,31,37,38,41}$ & 13 \\
Recreation and competitive $^{27,29,33}$ & 2 \\
College level $^{5,11,19}$ & 3 \\
School level $^{14}$ & 1 \\
Not stated/ not applicable $^{12,17,31}$ & 3 \\
\hline
\end{tabular}

\section{Matching criteria for subjects in case control studies}

Ten case control studies were identified. Only 1 of these studies included adolescents and all subjects in this study were uninjured. ${ }^{14}$ Five of the case control studies included injured and uninjured subjects. ${ }^{2,5,16,29,34}$ One author did not clearly indicate whether subjects were grouped or individually matched. ${ }^{29}$ Only 1 of these studies individually matched the injured and uninjured subjects. ${ }^{5}$ Individual matching is a more effective method of controlling for confounding because the effect of confounding factors is reduced and the results are therefore more robust. ${ }^{5}$

\section{Testing procedure}

The data collection procedures of published studies may require further scrutiny before extrapolating the results into practice. Sixteen of the 26 studies reviewed isolated the landing action by studying drop-jumping instead of the whole landing task (that also includes a take-off phase).

\section{Measurement tools}

One of the most common parameters assessed in studies analysing landing is ground reaction force (GRF), considered to be a risk factor for lower limb injury. ${ }^{29}$ Twenty-one of the studies $(80 \%)$ reviewed in this aspect of the research project utilised a force plate to measure GRFs.

Measurement of lower limb kinetics and kinematics using single cameras was done in 5 studies. One study used a tracking device and 1 used a potentiometer. An electrogoniometer was only utilised in 1 of the papers reviewed. Three-dimensional motion analysis equipment was used in 5 studies.

\section{Knee injury risk factors}

Landing with the knee in a more extended position (less than $45^{\circ}$ ) results in reduced energy absorption and may predispose individuals to injury. ${ }^{2,5,8,13,15,41}$ On the other hand, 1 study stated that deep knee flexion angles (more than $90^{\circ}$ ) when landing predisposed volleyball players to patellar tendonitis. ${ }^{34}$ It is important to note that there are differences in the method of knee angle measurement in the above studies and that this will influence the interpretation and clinical application of the results. The most appropriate knee flexion angle when landing and the relationship between the knee flexion angle and injury occurrence must therefore be explored further.

Eccentric knee control to maximise energy absorption is hypothesised to be critical to knee function when landing..$^{5,41}$ Eccentric knee control is thought to be important in maintaining knee stability as the knee joint flexes and the player has to control lowering the body. Poor dynamic stability will result in torsional forces at the knee joint, increasing injury risk of knee joint structures when landing. ${ }^{20}$

Large GRFs when landing are also postulated to increase injury risk. Landing height, reduced knee flexion, anterior tibial translation, vastus medialis activity, peak adduction and abduction moments and heel-toe landing are believed to result in relatively higher GRFs. ${ }^{1,8,14,29}$ Table VII presents a summary of knee injury risk factors.

\section{Discussion}

To the best of our knowledge this is the first systematic review of biomechanical knee injury risk factors when an individual lands from a jump. We searched the primary databases indexing biomechanical peer-review publications reporting on this topic. Date limits were set as the method- 


\section{TABLE VII. Knee injury risk factors identified}

Lower knee eccentric angular velocity is seen in patients with an old rupture of the $\mathrm{ACL}^{12}$

GRF increase with landing height and knee extension ${ }^{8}$

Landing in less knee flexion results in less energy absorption by the muscular system and more stress on the skeletal structures ${ }^{5}$

Magnitude of GRF is positively correlated to anterior tibial accelerations, increasing ACL strain ${ }^{29}$

Vastus medialis activity is an important predictor of $\mathrm{GRF}^{1}$

Peak adduction and abduction moments were predictors of $\mathrm{GRF}^{14}$

Deep knee flexion angles, high GRFs, rates of loading the extensor mechanisms and tibial external torsional moment predicted patellar tendonitis. ${ }^{34}$ Heel-toe landing results in higher GRF compared with toe-heel landing

Heel-toe landing leads to higher hip and knee negative power and work and less energy absorption by the plantarflexors ${ }^{19}$

The relationship between variability of selected joint moments and injury proneness is unclear ${ }^{16}$

Knee extensors are consistent contributors to energy dissipation across different landing techniques and heights. Eccentric strength of the knee extensors critical to knee function ${ }^{41}$

Effective instructions and auditory cues could reduce $\mathrm{GRF}^{28}$

Individuals with ankle instability compensate by increasing ankle dorsiflexion and knee flexion pre-and- post landing ${ }^{2}$

Initial flexion impact angle was reduced in females compared with males ${ }^{15}$

Activity post cryotherapy does not predispose to injury ${ }^{17}$

Augmented feedback can reduce landing forces ${ }^{31}$

ACL reconstructed group had reduced hip flexion, reductions in peak hip and knee negative power and reduced hip extensor energy absorption ${ }^{5}$

Reduced and poorly controlled knee flexion among females compared with males ${ }^{22}$

Verbal instructions increased knee flexion, but not earlier hamstring activation ${ }^{3}$

Under non-fatigued and fatigued conditions, no difference in knee flexion and muscle activity was seen between males and females ${ }^{11}$

Postpubescent females landed with the knee more extended, and had greater extensor moments and power that plays a role in increased risk of injury ${ }^{13}$

$\mathrm{ACL}=$ anterior cruciate ligament; $\mathrm{GRF}=$ ground reaction force.

ological quality of studies improved over time, especially over the mid-1990s. ${ }^{23}$

The review included a range of different study design types - 3 randomised controlled studies (11\%), 13 observational $(50 \%)$ and 10 case-control (39\%) studies. The methodological quality of the studies was only fair and it is thus difficult to draw conclusions regarding knee injury risk factors.

The generalisability of the study findings is compromised by unjustified sample sizes and sample recruitment methods. Sample sizes of less than 30 subjects are regarded as small since the power is substantially reduced and about $60 \%$ of the studies reviewed included 20 or fewer subjects. One reason for small sample sizes may be time-consuming practical implications when collecting and processing biomechanical data. Further technological improvements may reduce data collection and processing time, enabling researchers to recruit larger samples that can be representative of a population.

No study justified the reliability and validity of the measurement equipment. Establishing reliability of biomechanical equipment is a voluminous task and thus impractical for most researchers to undertake before they collect data. Establishing the reliability and validity of measurement tools may also not be within the research scope of research into clinical application of biomechanics. However it is important for researchers to understand the degree of reliability of the equipment by referring to published reports into reliability of their measurement tools.

Basketball arguably has the highest rate of knee injuries and may be one of the fastest growing sports in South
Africa. ${ }^{24}$ This review highlights the shortage of research into basketball to investigate biomechanical factors predisposing players to knee injuries. Each sport has specific characteristics and game dynamics and therefore further research to investigate biomechanical risk factors among basketball players is warranted.

This review of published biomechanical information since 1990 indicated no information regarding the landing strategies of knee-injured adolescents. Developmental stage influences neuromuscular control and consequently the landing strategies demonstrated by injured adolescents may differ from the technique demonstrated by injured adults. ${ }^{13}$ The landing strategies of uninjured adolescents are biomechanically less safe than those of uninjured adults. Adolescents presenting with a knee injury may further compromise joint stability when landing from a jump. Younger populations should thus be considered in future studies.

Most of the studies (61\%) isolated the analysis of landing from drop jumping. A recent study aimed to compare the landing phase of a drop-jump movement with a volleyball spike-jump movement among volleyball players. ${ }^{10}$ Results indicated that the 2 tasks differed significantly with respect to GRFs, lower limb kinematics and muscle activation synchrony. Therefore it may not be valid to modify the landing task to only the drop-landing phase and this must be considered when comparing results with those from studies that involved the whole landing task. Five (19\%) of the 26 studies reported sport-specific jumps. This highlights the lack of research into landing as a whole and the limitations for clinical application. 
This review indicated a wide range of biomechanical knee injury risk factors. Inadequate knee flexion angles when landing was nominated by $23 \%$ of the studies as a likely knee injury risk factor when landing. ${ }^{2,5,13,15,22,34}$ The degree of knee flexion may be an indication of the ability of the individual to control the eccentric knee movement and absorb shock, contributing towards joint stability and consequent injury prevention. ${ }^{23}$ However the variability in study samples and other methodological shortcomings hinders general acceptance among clinicians of knee flexion as a primary risk factor in knee injury.

The results of reviewed studies are potentially confounded by a number of factors. These include subject matching, subject recruitment method, and unclear inclusion and exclusion criteria. It is not possible to generalise those review findings and to establish relationships between biomechanical parameters and pathology in the presence of confounding factors illustrated by the studies reviewed.

According to Sackett's Evidence Hierarchy, the publications we reviewed provide level 2 a (11\% of studies) and level 3 ( $89 \%$ of studies) evidence regarding the biomechanical risk factors. Although high-level evidence is lacking for biomechanical knee injury risk factors when landing from a jump, the published research reviewed provides important information on causality and theories to direct well-designed future longitudinal and randomised studies.

\section{RefERENCES}

1. Caster B, Bates B. The assessment of mechanical and neuromuscular response strategies during landing. Med Sci Sports Exerc 1995; 27: 73644.

2. Caulfield B, Garret M. Functional instability of the ankle: Differences in patterns of ankle and knee movement prior to and post landing in a single leg jump. Int J Sports Med 2002; 23: 64-8.

3. Cowling E, Steele J, McNair P. Effect of verbal instructions on muscle activity and risk of injury to the anterior cruciate ligament injury during landing. Br J Sports Med 2003; 37: 126-30.

4. Crombie I. The Pocket Guide to Critical Appraisal: A Handbook for Health Care Professionals, London: BMJ Publishing Group, 1996.

5. Decker M, Torry M, Noonan T, Riviere A, Strerett W. Landing adaptations after ACL reconstruction. Med Sci Sports Exerc 2002; 34: 1408-13.

6. Devita P, Skelly W. Effect of landing stiffness on joint kinetics and energetics in the lower extremity. Med Sci Sports Exerc 1992; 24: 108-15.

7. Dufek J, Zhang S. Landing models for volleyball players. J Sports Med Phys Fitness 1996; 36: 35-42.

8. Dufek J, Bates B. The evaluation and prediction of impact forces during landings. Med Sci Sports Exerc 1990; 22: 370-7.

9. Dufek J, Bates B. Biomechanical factors associated with injury during landing in jump sports. Sports Med 1991; 12: 326-37.

10. Edwards S, Steele J. Is it valid to isolate the landing phase of a whole movement skill? Paper presented at Australian Conference of Science and Medicine in Sport, Melbourne, 12 - 16 October 2002.

11. Fagenbaum $R$, Darling $R$. Jump landing strategies in male and female college athletes and the implications of such strategies for anterior cruciate ligament injury. Am J Sports Med 2003; 31: 233 - 40.

12. Gauffin H, Petterson G, Tropp H. Kinematic analysis of one leg hopping in patients with an old rupture of the anterior cruciate ligament. Clinical Biomechanics 1990; 5: 41-6.

13. Hass C, Schick E, Chow J, Tillman M, Brunt D, Cauraugh J. Lower extremity biomechanics differ in prepubescent and post-pubescent female athletes during stride jump landings. Journal of Applied Biomechanics 2003; 19: $139-52$.
14. Hewett T, Stroupe A, Nance T, Noyes F. Plyometric training in female athletes. Decreased impact forces and hamstring torques. Am J Sports Med 1996; 24: 765-73.

15. Huston L, Vibert B, Ashton-Millee J, Wojtys E. Gender differences in knee angle when landing from a drop jump. Am J Knee Surg 2001; 14: 215-20.

16. James C, Dufek J, Bates B. Effects of injury proneness and task difficulty on joint kinetic variability. Med Sci Sports 2000; 32:1833-44.

17. Jameson A, Kinzey S, Hallam J. Lower extremity cryotherapy does not affect vertical ground reaction forces during landing. Journal of Sports Rehabilitation 2001; 10: 132-42.

18. Jones D, Louw Q, Grimmer K. Recreational and sporting injury to the adolescent knee and ankle: Prevalence and causes. Australian Journal of Physiotherapy 2000; 46: 179-88.

19. Kovacs I, Tihanyi J, Devita P, Recz L. Foot placement modifies kinematics and kinetics during drop-jumping. Med Sci Sports Exerc 1999; 31: 708-15.

20. Lephart S. Sensorimotor System. Performance and Protection. Paper presented at 7th Olympic Conference in Sports Sciences, Athens, 7-11 October 2003.

21. Lephart S. Sensorimotor system part 1. Physiological basis of joint stability. Journal of Athletic Training 2002; 37: 71-9.

22. Lephart S, Ferris C, Riemann B, Myers J, Fu F. Gender differences in strength and lower extremity kinematics during landing. Clin Orthop 2002; 401: 162-9.

23. Louw $\mathrm{Q}$. Knee injury prevention among adolescent basketball players $\mathrm{PhD}$ thesis, University of South Australia, 2004.

24. Louw Q, Grimmer K, Vaughan K. Knee injury patterns among young South African basketball players. South African Journal of Sports Medicine 2003; 15: 9-15.

25. McKay G, Goldie P, Payne W, Oakes B, Watson L. A prospective study of injuries in basketball: A total profile and comparison by gender and standard of competition. J Sci Med Sport 2001; 4: 196-211.

26. McNair P, Wood G, Marshall R. Stiffness of the hamstring muscles and relationship to function in anterior cruciate deficient individuals. Clinical Biomechanics 1992; 7: 131-7.

27. McNair P, Prapavessis $\mathrm{H}$. Normative data of vertical ground reaction forces during landing from a jump. J Sci Med Sport 1999; 2: 86-8.

28. McNair P, Prapavessis $\mathrm{H}$, Callender K. Decreasing landing forces. $\mathrm{Br} \mathrm{J}$ Sports Med 2000; 34: 293-6.

29. McNair P, Marshall R. Landing characteristics in subjects with normal and anterior cruciate ligament deficient knee joints. Arch Phys Med Rehabil 1994; 75: 584-9.

30. McNitt-Gray JL. Kinetics of lower extremities during drop landing from three heights. J Biomech 1993; 26: 1037-46.

31. Onate J, Guskiewicz K, Sullivan R. Augmented feedback reduces jump landing forces. J Orthop Sports Phys Ther 2001; 3: 511-7.

32. PEDro Scale. http://ptwww.cchs.usyd.edu.au/pedro (last accessed 1 June 2003).

33. Prapavessis $H$, McNair P. Effects of instruction in jumping technique and experience on ground reaction forces. J Orthop Sports Phys Ther 1999; 29: 352-6.

34. Richards D, Ajemian S, Wiley J, Zernicke R. Knee joint dynamics predict patellar tendonitis in elite volleyball players. Am J Sports Med 1996; 24: 676-83.

35. Riemann BL, Lephart S. Sensorimotor system: Part 1: Physiological basis of joint stability. J Ath Training 2002; 37: 71-9.

36. Sackett D, Richardson, Rosenberg W, Haynes R. Evidence Based Medicine. London: Oxford Churchhill Livingston, 2000.

37. Schot $P$, Barbara A, Mueller M. Within-participant variation in landing kinetics: Movement behaviour trait or transient? Res Q Exerc Sport 2002; 73: 450-6.

38. Schot $P$, Bates B, Dufek J. Bilateral performance symmetry during drop landing: A kinetic analysis. Med Sci Sports 1994; 26: 1153-9.

39. Steele J. Minimising lower limb injuries in sports. Paper presented at the Fifth IOC World Congress in Sport Sciences, Sydney, 31 October - 5 November 1999.

40. Van Mechelin W. Sports injury surveillance systems. Sports Med 1997; 24: 164-8.

41. Zhang S, Bates B, Dufek J. Contributions of lower extremity joints to energy dissipation during landings. Med Sci Sports Exerc 2000; 32: 812-9. 